Revue internationale de l'économie sociale

Recma

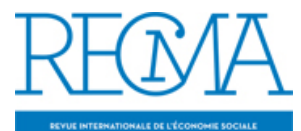

\title{
Le Circom et la revue Journal of rural cooperation
}

\section{Yair Levi}

Numéro 286, novembre 2002

URI : https://id.erudit.org/iderudit/1022244ar

DOI : https://doi.org/10.7202/1022244ar

Aller au sommaire du numéro

Éditeur(s)

Institut de l'économie sociale (IES)

\section{ISSN}

1626-1682 (imprimé)

2261-2599 (numérique)

Découvrir la revue

Citer ce compte rendu

Levi, Y. (2002). Compte rendu de [Le Circom et la revue Journal of rural cooperation]. Revue internationale de l'économie sociale, (286), 93-93.

https://doi.org/10.7202/1022244ar 
concurrence entre les initiatives privées et publiques de toutes sortes qui se multiplient sur ce terrain, poids du politique et du contexte économique - sont, comme on le voit tous les jours, de la plus grande actualité. On a cru à la Libération qu'un système généralisé de couverture sociale répondrait à l'ensemble des besoins de la population. La crise économique survenue depuis la fin des années 70 , la montée du chômage et de la pauvreté ont cruellement montré qu'il n'en était rien : ils expliquent le renouveau et la multiplication des dispositifs d'aide et d'assistance. Sur ce plan, Yannick Marec nous apporte de stimulants éléments de réflexion.

\section{Michel Dreyfus}

\section{Le Circom \\ et la revue Journal of rural cooperation}

C'est à Tel-Aviv en mars 1965, lors de la clôture d'un colloque international sur le rôle de la coopération dans le développement rural qui réunissait des représentants de dix-neuf pays, que fut décidée la création d'un centre international de recherche sur la coopération rurale. A l'initiative de feu les professeurs Henri Desroche de Paris, Ian Tauber de Prague et H. Darin-Drabkin de Tel-Aviv, le projet repose sur la mise en place d'un centre coordinateur à Paris, le Centre international de recherche sur le développement coopératif (Ircod), et de sous-divisions fonctionnelles reflétant la situation de l'époque: un centre à Prague pour la recherche sur l'agriculture collective, un centre au Mexique pour la recherche sur l'ejido ${ }^{(1)}$, et un centre à Tel-Aviv pour la recherche sur les communautés coopératives rurales. Le Centre international de recherche sur les communautés coopératives rurales (Circom) nait ainsi dans le "pays du kibboutz " en 1965, dans un contexte où bloc communiste et pays du tiersmonde jouent un rôle important.

La revue Journal of rural cooperation, publiée par le Circom, est créée en 1973 pour la diffusion d'études sur les coopératives et les communautés rurales. Une lecture des articles au fil du temps révèle un changement dans les préoccupations et les intérêts des auteurs. Si l'on s'intéresse aux trois premières et aux trois dernières années $(1973,1974$, 1975 et 2000, 2001, 2002, respectivement), on constate la diversité des enjeux auquels est confrontée, d'année en année, la coopération rurale. Parmi les vingt-cinq articles de la première catégorie, les sujets traités sont, par ordre d'importance: la justification d'une certaine intervention étatique dans les affaires des coopératives des pays du tiersmonde; la coopération comme moyen facilitant la transition entre le sous-développement et le monde du travail, de l'industrie et du « développement »; l'éloge de la théorie coopérative classique; le rôle du kibboutz et du moshav dans le développement rural; la recherche comparée dans les pays de l'Est européen et en Israël.

Tout cela change radicalement pendant les vingtcinq années suivantes. Au vu des vingt-huit articles de la deuxième catégorie, l'intérêt se porte désormais sur les coopératives de l'Ouest, puis sur la globalisation et ses effets. Viennent ensuite l'analyse de sujets peu connus comme: les coopératives d'agro-tourisme portées par les femmes en Grèce; la coopération au Groenland et en Corée du Nord; l'agriculture de groupe au Japon; la crise du kibboutz et la fin du moshav comme entité économique coopérative. Tout récemment, ce sont la position des coopératives et des communautés rurales vis-à-vis de l'économie sociale et une critique de l'orthodoxie coopérative classique qui ont fait l'objet d'articles.

A la situation initiale, caractérisée par ce que l'on pourrait appeler une certaine naïveté et des images stéréotypées, succède un processus de désenchantement et d'élargissement d'horizons; ce sont aujourd'hui de nouveaux problèmes que la revue s'efforce d'identifier, de saisir et de présenter à ses lecteurs.

\section{Yair Levi ${ }^{(2)}$}

(1) Terres concédées à des groupes paysans dans le cadre de la réforme agraire mexicaine (1934-1940), cf. Recma, n 248, 1993.

(2) Yair Levi est rédacteur en chef de la revue Journal of rural cooperation. Pour tout renseignement, s'adresser au Circom: Yad Tabenkin, RamatEfal 52960, Israël. Mél. : yalevi@netvision.net.il. 